\title{
МАТЕМАТИЧЕСКАЯ МОДЕЛЬ И АЛГОРИТМ РЕШЕНИЯ ЗАДАЧИ МАРШРУТИЗАЦИИ ТРАНСПОРТНЫХ СРЕДСТВ С НЕСКОЛЬКИМИ ЦЕНТРАМИ С ЧЕРЕДОВАНИЕМ И ЕДИНЫМ МЕСТОМ СБОРА
}

\author{
(C) 2021 С. Н. Медведев ${ }^{\bowtie}$ \\ Воронежский государственньй университет \\ Университетская пл., 1, 394018 Воронеж, Российская Федерация
}

\begin{abstract}
Аннотация. В статье рассматривается постановка задачи маршрутизации транспорта с несколькими центрами с чередованием и единым местом сбора. Для нее выделяются ключевые условия, которые отражают суть задачи и основные ограничения. С учетом этих условий представлена математическая модель задачи на основе специальным образом введенных булевых переменных, отвечающих за мини-маршрут. Также выделяются избыточные ограничения, которые могут следовать из постановки, но уже учтены в других ограничениях задачи. Для предложенной математической модели представлены теоремы об эквивалентных преобразованиях, которые необходимы для вычисления оценок множеств в ходе работы алгоритма. С использованием данных теорем строится точный алгоритм решения задачи на основе метода ветвей и границ. Представлено подробное описание шагов алгоритма. В завершении представлены результаты вычислительного эксперимента и их анализ. На основании полученных результатов можно сделать вывод об оптимальности найденных алгоритмом решений. Другой вывод заключается в необходимости доработки методики подсчета оценок множеств. Основными результатами исследования являются математическая модель и точный алгоритм решения задачи.
\end{abstract}

Ключевые слова: задача маршрутизации, дискретная модель, метод ветвей и границ, оценка множества.

\section{ВВЕДЕНИЕ}

Задача маршрутизации транспортных средств (ЗМТС) является расширением и модификацией задачи коммивояжера. Обе эти задачи являются NP-трудными [1]. Принципиальным отличием задачи маршрутизации от задачи коммивояжера является наличие специальной вершины, в которую можно возвращаться несколько раз. В литературе такую специальную вершину часто называют центром, базой или депо [2].

Классическая задача маршрутизации транспортных средств известна давно и хорошо изучена. Часто в задачах возникают

Медведев Сергей Николаевич e-mail: s_n_medvedev@mail.ru дополнительные ограничения и условия, которые приводят к новым постановкам, математическим моделям и алгоритмам решения. Выделяют 3МТС с несколькими типами транспортных средств с разной грузоподъемностью [3]. Известны открытая 3МТС, в которой транспортные средства могут не возвращаться на базу, задача маршрутизации транспортных средств с несколькими поездками, задача маршрутизации транспортных средств с возвратами и доставкой, ЗМТС с временными окнами, задача маршрутизации транспортных средств с дозагрузкой [3-7]. В [2] рассмотрена 3МТС, модель которой представлена с помощью графа, в котором клиенты (объекты) моделируются как ребра, а не как вершины. В [8] для постановки с двумя типами маршрутов строится смешанная целочисленная модель.

Контент доступен под лицензией Creative Commons Attribution 4.0 License.

The content is available under Creative Commons Attribution 4.0 License. 


\section{С. Н. Медведев}

Существуют различные методы решения как базовой, так и различных модификаций задачи маршрутизации транспортных средств. Известны методы, основанные на классических подходах к решению такого рода задач, а также специальные методы, отражающие некоторую оригинальную идею, применяемую к конкретной постановке.

К первой группе можно отнести методы, основанные на иерархической декомпозиции [7], на понятиях и алгоритмах теории графов [2], на матричных методах [3], на генетическом поиске $[9,10]$, на идеях отсечения и ветвления [8] и т. д.

Ко второй группе можно отнести методы, основанные на использовании эвристического «правила эллипса» [6], на создании гигантского маршрута с последующей его кластеризацией [2], на сбалансированном дихотомическом делении вершин на группы [11].

Не так много работ посвящено задаче маршрутизации с несколькими центрами [2]. В $[10,12,13]$ рассмотрена новая постановка такой задачи, в которой важным условием является обязательное чередование целевых объектов и центров в маршруте транспортного средства. В этих работах предложены приближенные алгоритмы решения задачи, основанные на генетических и муравьиных алгоритмах и жадных стратегиях.

В данной работе предлагается альтернативная математическая модель 3МТC с несколькими центрами с чередованием и единым местом сбора, основанная на специальном способе задания переменных, отвечающих за мини-маршрут. Также приводится теоретическое обоснование для построения точного алгоритма решения, основанного на методе ветвей и границ, и сам алгоритм.

\section{1. ПОСТАНОВКА ЗАДАЧИ}

Приведем постановку 3МТС с несколькими центрами с чередованием и единым местом сбора.

Есть совокупность неподвижных объектов (вершин) двух типов: целевые объекты (тип А) и центры (тип В). Известны затра- ты, необходимые для перемещения между объектами. Несколько мобильных объектов (транспортных средств) должны в совокупности посетить все неподвижные объекты типа А таким образом, чтобы суммарные затраты были минимальными. Причем целевые объекты и центры должны чередоваться в маршрутах транспортных средств. Кроме того, каждый целевой объект в совокупности можно посетить только один раз, а любой центр можно посетить сколько угодно раз. При этом все мобильные объекты начинают и заканчивают свой маршрут в некоторой фиксированной точке сбора.

Под затратами можно понимать расстояние, временные затраты, стоимость и т. п.

Содержательная постановка задачи маршрутизации с двумя типами объектов с чередованием может быть представлена как уборка стогов с поля в грузовые машины сельскохозяйственной техникой.

Пусть имеется поле, на котором расположены стога сена и некоторое количество грузовых машин. Также имеется некоторое количество тракторов. Известны расстояния между всеми стогами и машинами. Необходимо составить маршруты для тракторов так, чтобы все стога были погружены в машины, причем суммарно пройденный тракторами путь должен иметь минимальную длину. При этом необходимо учитывать, что все трактора начинают и заканчивают работу в одной точке сбора, а грузоподъемность тракторов, перевозящих стога, равна одному стогу [10].

Это одна из возможных содержательных постановок общей задачи маршрутизации транспортных средств с несколькими центрами с чередованием объектов. К другим практическим задачам, связанным с такой постановкой, можно отнести проблему дозаправок транспортных средств, задачу о доставке, работу многофункциональных логистических центров и т. д.

В данной статье будем придерживаться общей формулировки задачи и использовать следующие понятия: целевые объекты (или объекты типа А), центры (или объекты типа В), мобильные объекты (или транспортные сред- 
ства), место сбора. На рис. 1 целевые объекты обозначены кружками, центры -квадратами, а место сбора - прямоугольником.

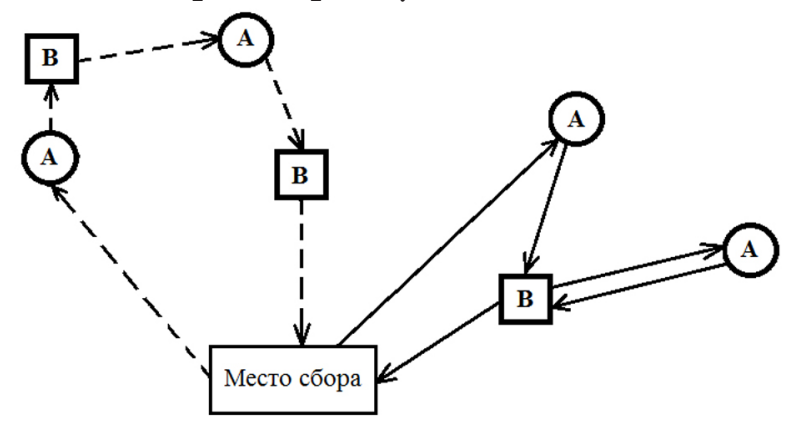

Рис. 1. ЗМТС с несколькими иентрами с чередованием и единым местом сбора

[Fig. 1. VRP with multiple centers with alternation and single gathering place]

\section{2. МАТЕМАТИЧЕСКАЯ МОДЕЛЬ}

Для составления математической модели поставленной задачи введем следующие обозначения:

$m$ - количество целевых объектов (неподвижных объектов типа А);

$n$ - количество центров (неподвижных объектов типа В);

$\left(c_{i j}\right)_{n \times m}-$ матрица, задающая затраты для перемещения между $i$-м центром (тип В) и $j$-м целевым объектом (тип А).

Введем переменные, отвечающие за мини-маршрут «B - A - B» (рис. 2). Таким образом, будет учтено требование чередования неподвижных объектов в маршруте транспортного средства. Альтернативные способы задания переменных представлены в $[10,13]$.

Пусть $\quad x_{i j k} \in\{0,1\}, \quad i=1, \ldots, n, \quad j=1, \ldots, m$, $k=1, \ldots, n$, причем $x_{i j k}=1$, если мобильный объект перемещается от $i$-го объекта типа В к $j$-му объекту типа А и перемещается от него к $k$-му объекту типа $\mathrm{B}$, и $x_{i j k}=0$ в противном случае.

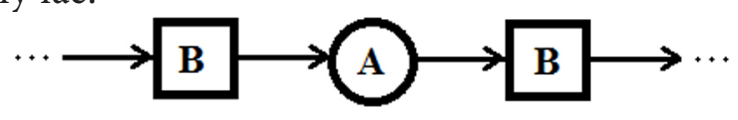

Рuс. 2. Мини-марирут

[Fig. 2. Mini route]

Также обозначим через $p$ количество мобильных объектов.

Учитывая введенные переменные, вычислим матрицу $\left(c_{i j k}\right)_{n \times m \times n}$, задающую суммар- ные затраты для перемещения между $i$-м центром, $j$-м целевым объектом и $k$-м центром, с элементами

$$
\begin{gathered}
c_{i j k}=c_{i j}+c_{k j}, i=1, \ldots, n, j=1, \ldots, m, \\
k=1, \ldots, n .
\end{gathered}
$$

Теперь необходимо определить такой объект как место сбора.

Чтобы итоговый маршрут каждого мобильного объекта был замкнутым, как и в задаче коммивояжера, необходимо, чтобы место сбора не нарушало требование чередования «... - А - В - А - В - ...». Если задать данный специальный объект как некоторый дополнительный объект типа А (рис. 3а)) или типа В (рис. 36)), то требование чередования нарушится.

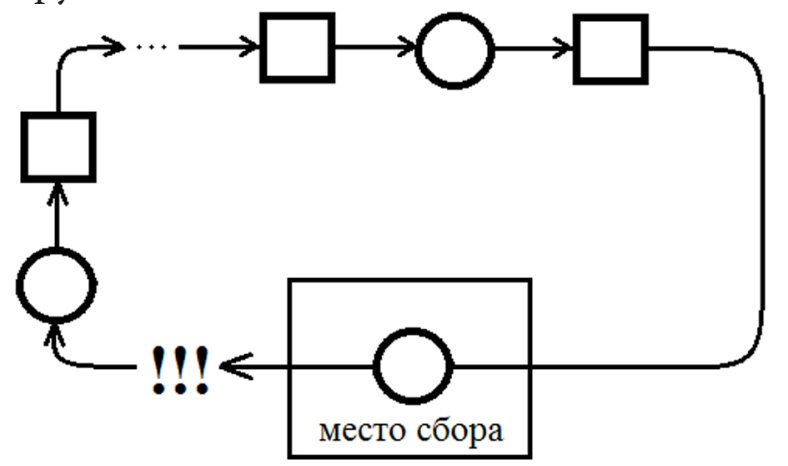

a)

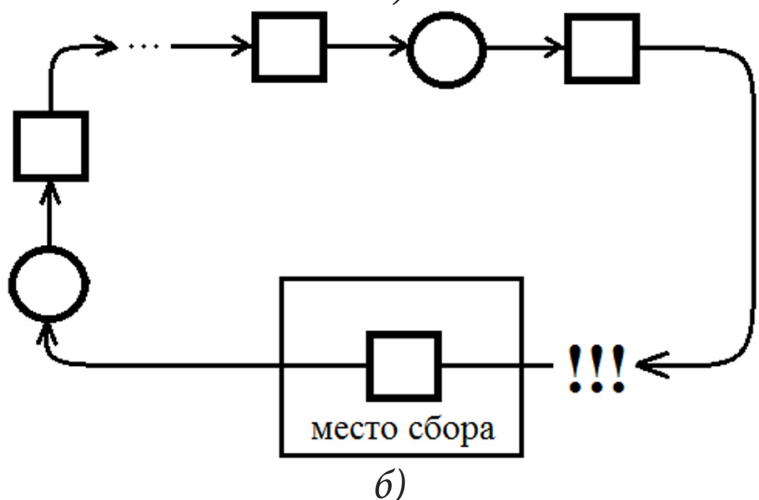

Рис. 3. а) место сбора - иелевой объект, б) место сбора - иентр

[Fig. 3. a) gathering place - target object, b) gathering place - center]

Тогда необходимо представить место сбора как совокупность двух фиктивных объектов типа А и типа В (рис. 4). Пусть этим объектам отвечают индексы $j=m+1$ и $i, k=0$ соответственно. Затраты для перемещения между фиктивными объектами примем равным нулю. Таким образом, получим матрицу 


\section{С. Н. Медведев}

$C=\left(c_{i j k}\right)_{(n+1) \times(m+1) \times(n+1)}, \quad i=0, \ldots, n, \quad j=1, \ldots, m+1$, $k=0, \ldots, n$.

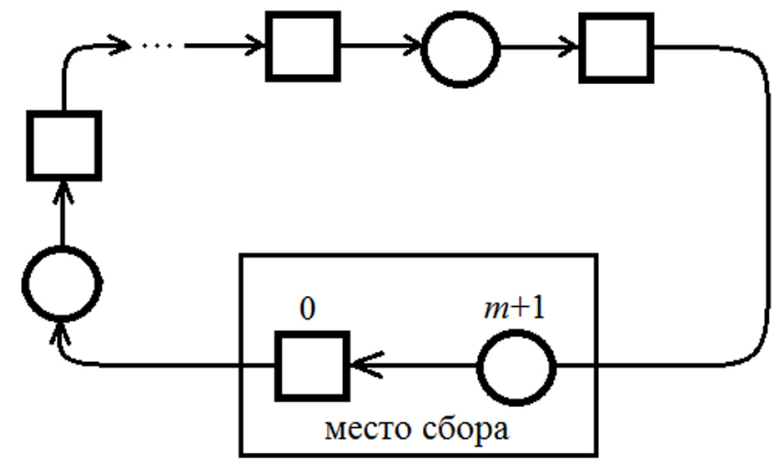

Puc. 4. Место сбора - пара объектов

[Fig. 4. Gathering place - pair of objects]

Составим математическую модель задачи, выделяя из постановки ключевые моменты, которые необходимы для конструирования ограничений задачи.

Условие 1. Совокупная длина маршрутов мобильных объектов должна быть минимальна.

Целевая функция задачи тогда запишется в виде

$$
\sum_{i=0}^{n} \sum_{j=1}^{m+1} \sum_{k=0}^{n} c_{i j k} x_{i j k} \rightarrow \min .
$$

Условие 2. К каждому целевому объекту должен приехать только один мобильный объект.

Условие 3. От каждого целевого объекта должен уехать только один мобильный объект.

Условия 2 и 3 запишутся одним ограничением вида

$$
\sum_{i=0}^{n} \sum_{k=1}^{n} x_{i j k}=1, j=1, \ldots, m .
$$

Заметим, что условия 2 и 3 не распространяются на фиктивный целевой объект.

Условие 4. Если к целевому объекту приезжает мобильный объект, то он же должен уехать от этого целевого объекта.

Условие 5. Если от целевого объекта отъезжает мобильный объект, то он же должен был приехать к этому целевому объекту.

Условия 4 и 5 выполняются за счет введенных переменных.

Условие 6. Если к центру приезжает мобильный объект, то он же должен уехать от этого центра.
Условие 7. Если от центра уезжает мобильный объект, то он же должен был приехать к этому центру.

По-другому условия 6 и 7 можно сформулировать в виде «сколько мобильных объектов к центру приезжает, столько же от него и уезжает». Они запишутся ограничением вида

$$
\sum_{i=0}^{n} \sum_{j=1}^{m+1} x_{i j k}=\sum_{i=0}^{n} \sum_{j=1}^{m+1} x_{k j i}, k=0, \ldots, n .
$$

Условие 8. Для каждого мобильного объекта типа С должен быть составлен связный маршрут.

Условие 8, которое является аналогом условия отсутствия подциклов в задаче коммивояжера [3], запишется следующим образом

$$
\begin{gathered}
u_{j}-u_{l}+N^{s}\left(\sum_{i=0}^{n} \sum_{k=0}^{n} \sum_{h=0}^{n} x_{i j k} x_{k l h}\right) \leq N^{s}-1, \\
j, l \in \tilde{J}^{s}, \quad j \neq l, \quad s=1, \ldots, p .
\end{gathered}
$$

Здесь $J^{s}$ - множество индексов целевых объектов, которые посетил $s$-й мобильный объект, $N^{s}$ - мощность множества $J^{s}, \tilde{J}^{s}-$ множество, полученное из множества $J^{s}$ удалением любого индекса; $u_{j}$ - переменные, принимающие произвольные вещественные значения. Можно показать [14], что их можно считать целыми неотрицательными. Например, удобно в качестве значений этих переменных брать порядковый номер объекта типа А в маршруте.

Также рассмотрим дополнительные условия, накладываемые на специальным образом введенный объект - место сбора.

Условие 9. Каждый мобильный объект должен приехать к фиктивному центру и уехать от него (рис. 5).

Так как за фиктивный центр отвечают индексы $i, k=0$, то получим следующие ограничения

$$
\begin{gathered}
\sum_{j=1}^{m+1} \sum_{k=0}^{n} x_{0 j k}=p, \\
\sum_{i=0}^{n} \sum_{j=1}^{m+1} x_{i j 0}=p .
\end{gathered}
$$

Данные ограничения повторяют ограничение (3), но в дополнение к нему, указывают точное количество посещений фиктивного центра. Одно из них является избыточным из-за ограничения (3). 


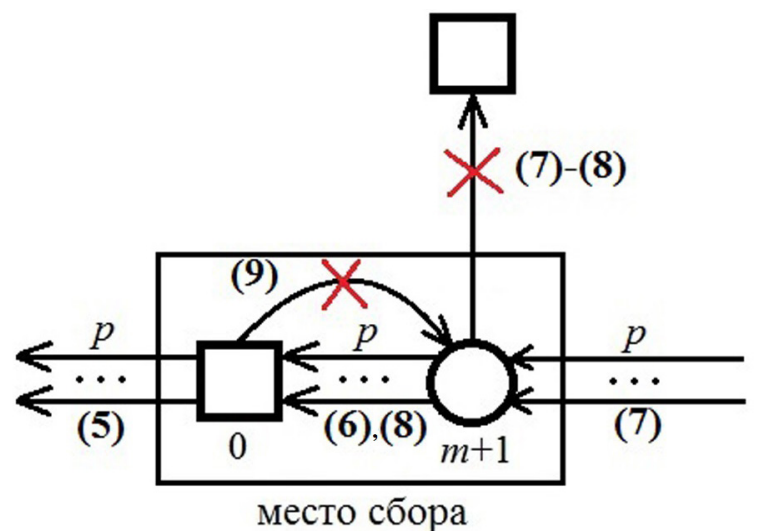

Рис. 5. Условия для фиктивной пары объектов [Fig. 5. Conditions for a fictitious pair of objects]

Условие 10. Каждый мобильный объект должен приехать к фиктивному целевому объекту и уехать от него (рис. 5).

Так как за фиктивный целевой объект отвечает индекс $j=m+1$, то получим следующее ограничение

$$
\sum_{i=0}^{n} \sum_{k=0}^{n} x_{i(m+1) k}=p .
$$

Условие 11. Все мобильные объекты должны от фиктивного целевого объекта приехать к фиктивному центру (рис. 5).

$$
\sum_{i=0}^{n} x_{i(m+1) 0}=p .
$$

Условие 12. Все мобильные объекты не могут от фиктивного центра уехать к фиктивному целевому объекту (рис. 5).

$$
\sum_{k=0}^{n} x_{0(m+1) k}=0 .
$$

Можно также рассмотреть Условие 13. Все мобильные объекты не должны уезжать от фиктивного целевого объекта ко всем центрам кроме фиктивного. Но это условие и логически, и математически выполняется за счет условий 10 и 11 и является избыточным. Действительно, из ограничения (7) получим

$$
\begin{aligned}
& \sum_{i=0}^{n} \sum_{k=0}^{n} x_{i(m+1) k}=p, \\
& \sum_{i=0}^{n} \sum_{k=1}^{n} x_{i(m+1) k}+\sum_{i=0}^{n} x_{i(m+1) 0}=p .
\end{aligned}
$$

Подставляя в последнее равенство ограничение (8), получим

$$
\sum_{i=0}^{n} \sum_{k=1}^{n} x_{i(m+1) k}+p=p,
$$

откуда

$$
\sum_{i=0}^{n} \sum_{k=1}^{n} x_{i(m+1) k}=0,
$$

что и является математической формализацией условия 13.

В итоге получена математическая модель (1)-(9) 3МТС с несколькими центрами с чередованием и единым местом сбора.

Таким образом, поставленная задача представляет собой задачу дискретной оптимизации, содержащую булевы переменные.

\section{3. АЛГОРИТМ РЕШЕНИЯ}

Для полученной математической модели 3МТС с несколькими центрами с чередованием и единым местом сбора представим точный алгоритм решения на основе метода ветвей и границ. Для этого необходимо определить, как будет осуществляться ветвление, как будут вычисляться оценки множеств, и какова будет стратегия обхода.

Ветвление, как и во многих задачах булевой оптимизации, будет основываться на разделении допустимого множества решений на два, в одном из которых значение рассматриваемой переменной $x_{i j k}$ равно единице, а в другом - нулю.

Стратегия обхода дерева может быть выбрана любой, но для конкретной реализации остановимся на следующей: вниз по дереву по левой стороне, вверх по дереву - до ближайшей незакрытой ветки.

Остановимся подробнее на вычислении оценки для текущего множества, так как эта часть метода всегда тесно связана с рассматриваемой задачей.

Доказаны следующие теоремы.

Теорема 1. Если элементы матрии $\left(c_{i j k}\right)_{(n+1) \times(m+1) \times(n+1)} u \quad\left(d_{i j k}\right)_{(n+1) \times(m+1) \times(n+1)} \quad$ связанbl равенствами

$$
d_{i j k}=c_{i j k}+\beta_{j}
$$

для всех $j=1, \ldots, m$ при фиксированных $i, k=0, \ldots, n$, то ЗМТС с несколькими иентрами с чередованием объектов и единым местом сбора с данными матрицами эквивалентны, то есть множества их оптимальных решений совпадают. Значения иелевых 


\section{С. Н. Медведев}

функиий данных задач отличаются на величину $\sum_{j=1}^{m} \beta_{j}$.

Теорема

$\left(c_{i j k}\right)_{(n+1) \times(m+1) \times(n+1)} u \quad\left(d_{i j k}\right)_{(n+1) \times(m+1) \times(n+1)}$

матрии связаны равенствами

$$
d_{i(m+1) k}=c_{i(m+1) k}+\beta_{(m+1)},
$$

для всех $i, k=0, \ldots, n$, то ЗМТС с несколькими иентрами с чередованием объектов и единым местом сбора с данными матрицами эквивалентны, а значения изелевых функиий данных задач отличаются на величину $p \beta_{m+1}$, где $p=\sum_{i=0}^{n} \sum_{k=0}^{n} x_{i(m+1) k}$.

\section{Теорема 3. Если элементы} $\left(c_{i j k}\right)_{(n+1) \times(m+1) \times(n+1)} u \quad\left(d_{i j k}\right)_{(n+1) \times(m+1) \times(n+1)}$

матрич, связаны равенствами

$$
\begin{aligned}
& d_{i j k}=c_{i j k}+\alpha_{i}, \\
& d_{k j i}=c_{k j i}+\gamma_{i},
\end{aligned}
$$

для всех $j=1, \ldots, m+1, k=0, \ldots, n, n р и$ фиксированном $i=0, \ldots, n$, то ЗМТС с несколькими иентрами с чередованием объектов и единым местом сбора с данными матрицами эквивалентны, а значения иелевых фбункиий данньх задач совпадают, при условии, что $\alpha_{i}=-\gamma_{i}$, $i=0, \ldots, n$.

В данных теоремах $\alpha_{i}, \beta_{j}, \gamma_{i}-$ произвольные числа, $j=1, \ldots, m+1, i, k=0, \ldots, n$.

Преобразования (10)-(13) будем называть эквивалентными преобразованиями для задачи (1)-(9).

Учитывая теоремы 1 и 2, всегда можно считать, что элементы матрицы $C$ являются неотрицательными. Действительно, выбрав в качестве $\beta_{j}=-\min _{i, k=0, \ldots, n}\left(c_{i j k}\right), \quad j=1, \ldots, m+1 \quad$ и применив преобразования (10), (11), получим, что все $c_{i j k} \geq 0, j=1, \ldots, m+1, i, k=0, \ldots, n$. Такую процедуру назовем приведением матрицы, а константы $\beta_{j}, j=1, \ldots, m+1$ - при-

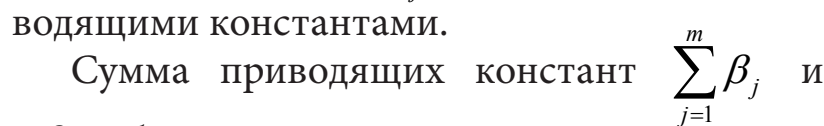
$p \beta_{m+1}$ будут использоваться в алгоритме для подсчета оценок множеств.

В алгоритме будем использовать следующие обозначения:

$i_{\text {_ }}$ cur - центр, из которого будет совершен выезд,

$J$ - множество индексов целевых объектов, $\xi$ - значение оценки,

$R$ - значение рекорда,

tabu - булева переменная, значение которой равно единице только тогда, когда на прошлой итерации было запрещено посещение фиктивного целевого объекта.

Приведем подробное описание алгоритма.

\section{Алгоритм.}

Этап 0. Инициализация.

\section{1. Задать}

$i_{\text {_cur }}=0, R=0, J=\{1, \ldots, m+1\}$,

$\varphi_{m+1}=0$, tabu $=1$.

Этап 1. Приведение матрицы по слоям $j=1, \ldots, m+1$.

1. Найти

$\beta_{j}=\min _{i, k=0, \ldots, n}\left(c_{i j k}\right), j=1, \ldots, m+1$.

2. Привести матрицу

$c_{i j k}=c_{i j k}-\beta_{j}, \quad j=1, \ldots, m+1, \quad i=0, \ldots, n$, $k=0, \ldots, n$.

3. Посчитать оценку

$$
\xi=\sum_{j=1}^{m+1} \beta_{j} \text {. }
$$

Этап 2. Выбор пути.

1. Проверить, есть ли запрет на заезд в фиктивный целевой объект: $t a b u=1$ ?

Если нет, то выбрать минимальный элемент

$$
c_{\left(i_{-} \text {cur }\right) j^{\prime} k^{\prime}}=\min _{j \in J, k=0, \ldots, n}\left(c_{\left(i_{-} c u r\right) j k}\right) .
$$

Если да, то выбрать минимальный элемент без учета $j=m+1$ и без учета $k=0$

$$
c_{\left(i_{-} c u r\right) j^{\prime} k^{\prime}}=\min _{j \in J\{\{m+1\}, k=1, \ldots, n}\left(c_{\left(i_{-} c u r\right) j k}\right),
$$

снять запрет $t a b u=0$.

2. Ветвление.

2.1. Ветка $x_{\left(i_{-} \text {cur }\right)^{\prime} k^{\prime}}=1$, то есть в маршрут выбран путь «центр с номером $i_{-}$cur - целевой объект $j^{\prime}$ - центр $k^{\prime} »$.

1. Пересчитать оценку

$$
\xi=\xi+c_{\left(i_{-} \text {cur }\right) j^{\prime} k^{\prime}} .
$$

2. Проверить, является ли выбранный целевой объект фиктивным: $j^{\prime}=m+1$ ?

Если да, то пересчитать оценку

$$
\xi=\xi+\beta_{m+1},
$$

если нет, то убрать целевой объект $j^{\prime}$ из рассмотрения

$$
J=J \backslash\left\{j^{\prime}\right\}
$$


3. Изменить $i_{-}$cur

$$
i_{-} \text {cur }=k^{\prime} \text {. }
$$

4. Проверить, посещены ли все объекты типа А: $J=\{m+1\}$ ?

Если да, то перейти к этапу 3 ,

если нет, то перейти к этапу 2.

2.2. Ветка $x_{(\text {i_cur }) j^{\prime} k^{\prime}}=0$, то есть соответствующий путь не выбран в маршрут.

1. Проверить, является ли объект типа А фиктивным: $j^{\prime}=m+1$ ?

Если да, то убрать его из рассмотрения на следующей итерации: $t a b u=1$,

если нет, то исключить возможность вы-

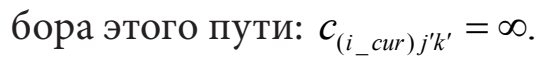

2. Привести матрицу и обновить оценку.

$$
\begin{gathered}
\beta_{j^{\prime}}=\min _{i, k=0, \ldots, n}\left(c_{i j^{\prime} k}\right), j=1, \ldots, m, \\
c_{i j^{\prime} k}=c_{i j^{\prime} k}-\beta_{j^{\prime}}, i=0, \ldots, n, k=0, \ldots, n, \\
\xi=\xi+\beta_{j^{\prime}} .
\end{gathered}
$$

3. Перейти к этапу 2.

Этап 3. Обновление рекорда.

1. Замкнуть маршрут

$$
x_{\left(i_{-} \text {cur }\right)(m+1) 0}=1 .
$$

2. Обновить оценку

$$
\xi=\xi+c_{\left(i_{-} \text {cur }\right)(m+1) 0} .
$$

3. Обновить рекорд.

Проверить: $\xi>R$ ?

Если да, то обновить рекорд $R=\xi$,

если нет, то рекорд не менять.

На этапе инициализации необходимо внести дополнительные изменения в матрицу $C$ в соответствии с ограничениями задачи (5)(9). А именно, элементы $c_{0(m+1) k}, k=0, \ldots, n$, $c_{i(m+1) k}, i, k=1, \ldots, n, \quad c_{i j 0}, j=1, \ldots, m, i=0, \ldots, n$ необходимо приравнять к некоторому большому числу или бесконечности, чтобы алгоритмически запретить выбор этих элементов на этапе приведения матрицы и на этапе выбора пути.

Заметим, что на первом шаге Этапа 2 выбранный элемент $c_{\left(i_{-} \text {cur }\right)^{\prime} k^{\prime}}$ не всегда равен нулю, так как приведение на первом этапе производится только по слоям $j$ согласно теоремам 1 и 2. В связи с этим на первом шаге ветки 2.1 производится пересчет оценки.

\section{4. РЕЗУЛЬТАТЫ И ИХ ОБСУЖДЕНИЕ}

Для анализа построенного алгоритма была разработана его программная реализация. Проведена серия вычислительных экспериментов со следующими входными и выходными данными.

Входные данные: матрицы расстояний для задач разной размерности.

Расстояния вычислялись на основании случайного равномерного распределения целевых объектов (тип А) и центров (тип В) на квадратной плоскости размера $100 \times 100$.

Для каждого эксперимента расчеты проводились на 500 матрицах.

Выходные данные: среднее арифметическое значений целевой функции, среднее арифметическое количества ветвлений, среднее арифметическое времени работы алгоритма.

В табл. 1 представлены результаты экспериментов.

Таблица 1. Результаты эксперимента [Table Experiment results]

\begin{tabular}{|c|c|c|c|}
\hline $\begin{array}{c}\text { тип В× } \\
\text { тип А }\end{array}$ & $\begin{array}{c}\text { Значение } \\
\text { ц.ф. }\end{array}$ & $\begin{array}{c}\text { Кол-во } \\
\text { ветвлений }\end{array}$ & $\begin{array}{c}\text { Время, } \\
\text { мс }\end{array}$ \\
\hline $1 \times 10$ & 1087 & 59 & $<1$ \\
\hline $1 \times 30$ & 2983 & 456 & 10 \\
\hline $1 \times 50$ & 4923 & 1128 & 45 \\
\hline $1 \times 100$ & 9768 & 4596 & 373 \\
\hline $2 \times 10$ & 874 & 3328 & 59 \\
\hline $2 \times 30$ & 1878 & 15657 & 1032 \\
\hline $2 \times 50$ & 3062 & 60853 & 1793 \\
\hline $2 \times 100$ & 6041 & $>200000$ & $>15000$ \\
\hline $5 \times 10$ & 605 & 92118 & 6274 \\
\hline $5 \times 30$ & 1535 & $>200000$ & $>20000$ \\
\hline $5 \times 50$ & 2388 & $>300000$ & $>20000$ \\
\hline $5 \times 100$ & 4705 & $>300000$ & $>30000$ \\
\hline $10 \times 10$ & 565 & $>300000$ & $>30000$ \\
\hline $10 \times 30$ & 1119 & $>300000$ & $>50000$ \\
\hline $10 \times 50$ & 1697 & $>400000$ & $>60000$ \\
\hline $10 \times 100$ & 3320 & $>400000$ & $>100000$ \\
\hline
\end{tabular}

По результатам проведенного эксперимента можно сказать, что количество объек- 


\section{C. Н. Медведев}

тов, в которые можно возвращаться неограниченное число раз (центры, тип В), намного больше влияет на время работы алгоритма и на число ветвлений. В то же время увеличение количества объектов типа В сокращает значение целевой функции, что можно увидеть и в табл. 1, и на графике (рис. 6). График построен для 10 целевых объектов, на горизонтальной оси отмечено количество центров, на вертикальной оси - средние значения целевой функции, взятые из табл. 1.

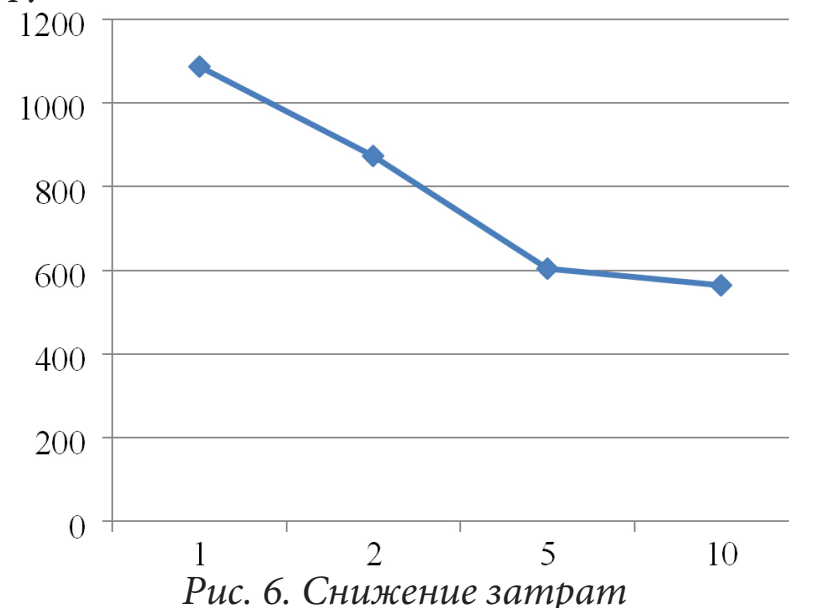

[Fig. 6. Cost reduction]

В табл. 2 отражены результаты ещё одной серии экспериментов с дополнительным числом объектов типа В, который также показывает рост числа ветвлений при увеличении количества центров.

Таблица 2. Увеличение количества объектов типа $B$

[Table 2. Increase in the number of objects B type]

\begin{tabular}{|c|c|}
\hline тип В×тип А & Кол-во ветвлений \\
\hline $1 \times 10$ & 59 \\
\hline $2 \times 10$ & 3328 \\
\hline $3 \times 10$ & 12982 \\
\hline $4 \times 10$ & 70719 \\
\hline $1 \times 30$ & 456 \\
\hline $2 \times 30$ & 15657 \\
\hline $3 \times 30$ & 117804 \\
\hline $4 \times 30$ & $>200000$ \\
\hline
\end{tabular}

На рис. 7 построен график для 10 целевых объектов, на горизонтальной оси отмечено количество центров, на вертикальной оси средние значения числа ветвлений, взятые из табл. 2.

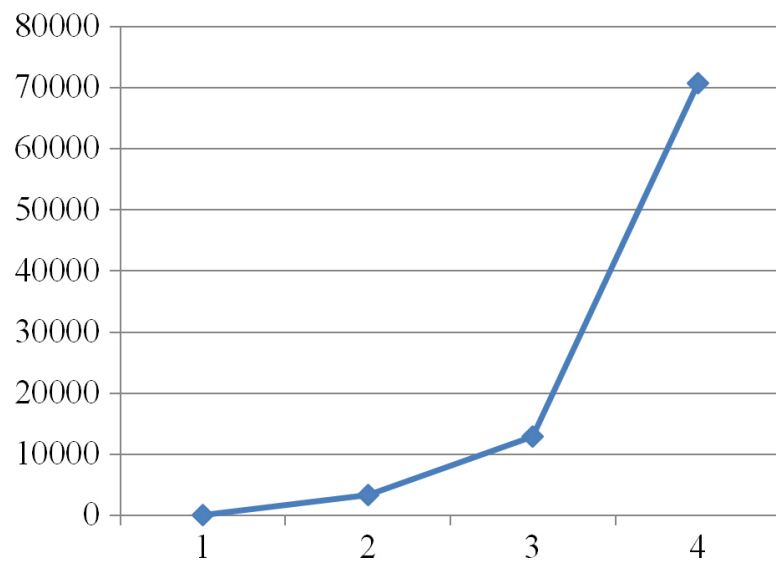

Рис. 7. Увеличение числа ветвлений

[Fig. 7. Increase of branching]

По результатам данных экспериментов следует сделать вывод о том, что предложенный способ вычисления оценки должен быть доработан. Большое количество ветвлений говорит о том, что во многих узлах оценка меньше рекорда, поэтому ветки не «закрываются», и ветвление продолжается. Следует улучшить методику подсчета оценок, возможно, используя теорему 3, которая на данном этапе исследования никак не задействована в алгоритме.

Следующая серия экспериментов нацелена на вычислительное подтверждение того, что алгоритм находит оптимальное решение. Для известных матриц расстояний с одним центром добавлены расстояния от всех целевых объектов до ещё одного центра, который заведомо расположен в худшей позиции по сравнению с имеющимся.

Проиллюстрируем данный эксперимент на примере. Дана матрица расстояний, в первой строке которой записаны расстояния от фиктивного центра до целевых объектов (в том числе фиктивного), а во второй строке расстояние от основного центра до всех целевых объектов.

$$
\left(\begin{array}{cccc}
17 & 12 & 9 & 0 \\
5 & 4 & 6 & 12
\end{array}\right)
$$

Добавим ещё один центр, который расположен заведомо хуже имеющегося. В матрице появится третья строка.

$\left(\begin{array}{cccc}17 & 12 & 9 & 0 \\ 5 & 4 & 6 & 12 \\ 111 & 107 & 121 & 131\end{array}\right)$


Очевидно, что оптимальное решение должно сохраниться, так как обязательного требования заезда во все центры нет. То есть добавленный центр должен остаться не посещенным.

Для проверки этого факта был проведен эксперимент. Были взяты матрицы расстояний для случая, когда один центр располагается в центре области, а целевые объекты равномерно распределены по ней. Для таких матриц были посчитаны значения целевой функции и количество ветвлений. После был введен дополнительный центр, расположенный заведомо далеко. И вычисления были проведены для обновленных матриц.

Результаты эксперимента представлены в табл. 3.

Таблица 3. Дополнительный объект типа $B$ [Table 3. Additional object B type]

\begin{tabular}{|c|c|c|}
\hline тип В×тип А & Значение ц.ф. & $\begin{array}{c}\text { Кол-во } \\
\text { ветвлений }\end{array}$ \\
\hline $1 \times 10$ & 1068 & 28 \\
\hline $2 \times 10$ & 1068 & 29 \\
\hline $1 \times 30$ & 2883 & 141 \\
\hline $2 \times 30$ & 2883 & 166 \\
\hline $1 \times 50$ & 4655 & 295 \\
\hline $2 \times 50$ & 4655 & 363 \\
\hline $1 \times 100$ & 9107 & 1136 \\
\hline $2 \times 100$ & 9107 & 1447 \\
\hline
\end{tabular}

Из табл. 3 видно, что значение целевой функции не меняется, но количество ветвлений увеличилось при введении дополнительного центра. Таким образом, можно сделать вывод, что алгоритм работает правильно и действительно получает оптимальное решение.

\section{ЗАКЛЮЧЕНИЕ}

Для задачи маршрутизации транспортных средств с несколькими центрами с чередованием и единым местом сбора построена математическая модель в виде задачи дискретной оптимизации. Отличием данной задачи от других известных формулировок ЗМТС является требование чередования двух типов объектов с разными свойствами в маршруте транспортного средства. Основываясь на теоремах об эквивалентных задачах и схеме метода ветвей и границ, разработан точный алгоритм решения задачи. Проведенный эксперимент показал достоинства и проблемы разработанного алгоритма. Дальнейшие исследования должны быть направлены на модификацию способа приведения матриц для более эффективного вычисления оценок.

\section{КОНФЛИКТ ИНТЕРЕСОВ}

Автор декларирует отсутствие явных и потенциальных конфликтов интересов, связанных с публикацией настоящей статьи.

\section{СПИСОК ЛИТЕРАТУРЫ}

1. Lenstra, J. K. Complexity of vehicle routing and scheduling problems / J. K. Lenstra, A. H. G. Kan // Networks. - 1981. - V. 11, No 2. P. 221-227. DOI: 10.1002/net.3230110211.

2. Amberg, A. Multiple center capacitated arc routing problems : A tabu search algorithm using capacitated trees / A. Amberg, W. Domschke, S. Voss // European Journal of Operational Research. - 2000. - V. 124. - P. 360-376. DOI:10.1016/S0377-2217(99)00170-8.

3. Ижков, С. А. Матричный подход в решении задачи маршрутизации с несколькими транспортными средствами / С. А. Ишков, Е. С. Ишкова // Известия Самарского научного центра Российской Академии Наук. 2011. - T. 13, № 4. - C. 189-164.

4. Ekici, A. Cyclic Delivery Schedules for an Inventory Routing Problem / A. Ekici, O. Özener, G. Kuyzu // Transportation Science. 2015. - V. 49, No 4. - P. 817-829. DOI: 10.1287/ trsc.2014.0538.

5. I-Ming, Ch. The Team Orienteering Problem / I-Ming Chao, B. Golden and E. Wasil // European Journal of Operational Research. 1996. - 88, No 3. - P. 464-474. DOI:10.1016/03772217(94)00289-4.

6. Santos, L. An improved heuristic for the capacitated arc routing problem / L. Santos, J. Coutinho-Rodriguesa, J. R. Currentb // Com- 


\section{C. Н. Медведев}

puters and Operations Research. - 2009. - V. 6. P. 2632-2637. DOI:10.1016/j.cor.2008.11.005.

7. Tang, K. Scalable Approach to Capacitated Arc Routing Problems Based on Hierarchical Decomposition / K. Tang, J. Wang, X. Li and X. Yao // Research of Birmingham. - University of Birmingham. - 2015. - P. 15. DOI: 10.1109/ TCYB.2016.2590558.

8. Dominguez-Martín, B. The driver and vehicle routing problem / B. Domínguez-Martín, I. Rodríguez-Martín, J.-J. Salazar-González // Computers \& Operations Research. - 2018. V. 92. - P. 56-64. DOI: 10.1016/j.cor.2017.12.010.

9. Григорьев, В. П. Маршрутизация доставки розничной продукции в городской дорожной сети на основе генетического алгоритма / В. П. Григорьев, К. А. Киселев // Известия Томского политехнического университета. 2007. - T. 310, № 2. - C. 195-199.

10. Medvedev, S. The vehicle routing problem for several agents among the objects of two types / S. Medvedev, A. Sorokina, O. Medvedeva // XXI International Conference Complex Systems: Control and Modeling Problems (CSCMP). Samara. - 2019. - P. 535-540. DOI: 10.1109/ CSCMP45713.2019.8976624.
11. Пожидаев, М. С. Алгоритмы решения задачи маршрутизации транспорта : автореф... дис. канд. техн. наук. - Томск : ТГУ, 2010. - 16 c.

12. Medvedev, S. Comparative analysis of the exact and heuristic algorithms for solving the vehicle routing problem for several agents among the objects of two types // 2nd International Conference on Control Systems, Mathematical Modeling, Automation and Energy Efficiency (SUMMA). - 2020. - Lipetsk. - P. 817-822. DOI: 10.1109/SUMMA50634.2020.9280609

13. Medvedev, S. N. Formulation and algorithmization of the interleaved vehicle routing problem / S. N. Medvedev, O. A. Medvedeva, Y. R. Zueva, G. D. Chernyshova // Journal of Physics: Conference Series. - Institute of Physics Publishing. - 2019. - V. 1203, No 012053. - P. 15. DOI: $10.1088 / 1742-6596 / 1203 / 1 / 012053$.

14. Корбут, А. А. Дискретное программирование / А. А. Корбут, Ю. Ю. Финкельштейн [под ред. Д. Б. Юдина]. - Москва : Наука, 1969. - 368 c.

Медведев Сергей Николаевич - канд. физ.-мат. наук, доц., доцент кафедры вычислительной математики и прикладных информационных технологий факультета прикладной математики, информатики и механики Воронежского государственного университета.

E-mail: s_n_medvedev@mail.ru

ORCID iD: https://orcid.org/0000-0002-6971-5069 


\title{
A MATHEMATICAL MODEL AND AN ALGORITHM FOR SOLVING A MULTI- DEPOT VEHICLE ROUTING PROBLEM WITH A SINGLE END POINT
}

\author{
(C) 2021 S. N. Medvedev $\bowtie$ \\ Voronezh State University \\ 1, Universitetskaya Square, 394018 Voronezh, Russian Federation
}

\begin{abstract}
Annotation. The article considers a multi-depot vehicle routing problem with a single end point. First, the key conditions reflecting the nature of the problem and the main constraints are determined. These conditions are used to create a mathematical model of the problem based on specially introduced Boolean variables specifying the mini-route. We also determine the redundant constraints, which may follow from the formulation of the problem, but have already been taken into account in other constraints. The article presents equivalent transformation theorems for the suggested mathematical model. Those theorems are necessary to calculate the estimates of the sets at each iteration of the algorithm. The theorems are also used to design an exact algorithm for solving the problem based on the branch-and-bound method. The article details each step of the algorithm. It also presents and analyses the results of a computational experiment. The results demonstrate that the algorithm managed to find optimal solutions. We also came to the conclusion that it is necessary to refine the methodology for calculating the set estimates. The key results of the study are the mathematical model and the exact algorithm for solving the problem. Keywords: vehicle routing problem, discrete model, branch-and-bound method, set estimation.
\end{abstract}

\section{CONFLICT OF INTEREST}

The author declare the absence of obvious and potential conflicts of interest related to the publication of this article.

\section{REFERENCES}

1. Lenstra J. K. \& Kan H. G. (1981) Complexity of vehicle routing and scheduling problemsm // Networks. 11(2). P. 221-227. Available from: DOI: $10.1002 /$ net.3230110211.

2. Amberg A., Domschke W. \& Voss S. (2000) Multiple center capacitated arc routing problems : A tabu search algorithm using capacitated trees // European Journal of Operational Research. 124. P. 360-376. Available from: DOI:10.1016/S03772217(99)00170-8.

3. Ishkov S. A. \& Ishkova E. S. (2011) Matrix approach in solving the routing problem with several vehicles // Bulletin of the Samara Scien-

Medvedev Sergey N.

e-mail: s_n_medvedev@mail.ru tific Center of the Russian Academy of Sciences. 13(4). P. 189-164.

4. Ekici A., Özener O. \& Kuyzu G. (2015) Cyclic Delivery Schedules for an Inventory Routing Problem // Transportation Science. 49(4). P. 817-829. Available from: DOI: 10.1287/ trsc.2014.0538.

5. I-Ming Ch., Golden B. \& Wasil E. (1996) The Team Orienteering Problem // European Journal of Operational Research. 88(3). P. 464-474. Available from: DOI:10.1016/0377-2217(94)00289-4.

6. Santos L., Coutinho-Rodriguesa J. \& Currentb J. R. (2009) An improved heuristic for the capacitated arc routing problem // Computers and Operations Research. 6. P. 2632-2637. Available from: DOI:10.1016/j.cor.2008.11.005.

7. Tang K., Wang J., Li X. \& Yao X. (2017) A Scalable Approach to Capacitated Arc Routing Problems Based on Hierarchical Decomposition // in IEEE Transactions on Cybernetics. 47(11). P. 3928-3940. Available from: DOI: 10.1109/TCYB.2016.2590558.

8. Domínguez-Martín B., Rodríguez-Martín I. \& Salazar-González J.-J. (2018) The driver and 


\section{С. Н. Медведев}

vehicle routing problem // Computers \& Operations Research. 92. P. 56-64. Available from: DOI: $10.1016 /$ j.cor.2017.12.010.

9. Grigoriev V. P. \& Kiselev K. A. (2007) Routing of delivery of retail products in the urban road network based on a genetic algorithm // Bulletin of the Tomsk Polytechnic University. 310(2). P. 195-199. (in Russian)

10. Medvedev S., Sorokina A. \& Medvedeva O. (2019) The vehicle routing problem for several agents among the objects of two types // In: XXI International Conference Complex Systems: Control and Modeling Problems, CSCMP 2019, 3-6 September 2019, Samara, Russia. P. 535-540. Available from: DOI: 10.1109/CSCMP45713.2019.8976624.

11. Pozhidaev M. S. (2010) Algorithms for solving the problem of routing transport. Abstract $\mathrm{PhD}$ thesis. Tomsk State University. (in Russian)
12. Medvedev S. (2020) Comparative analysis of the exact and heuristic algorithms for solving the vehicle routing problem for several agents among the objects of two types // In: 2nd International Conference on Control Systems, Mathematical Modeling, Automation and Energy Efficiency, SUMMA 2020, 11-13 November 2020, Lipetsk, Russia. P. 817-822. Available from: DOI: 10.1109/SUMMA50634.2020.9280609

13. Medvedev S. N., Medvedeva O. A., Zueva Y. R. \& Chernyshova G. D. (2019) Formulation and algorithmization of the interleaved vehicle routing problem // Journal of Physics: Conference Series. 1203 (012053), 15. Available from: DOI: 10.1088/1742-6596/1203/1/012053.

14. Korbut A. A. \& Finkelstein Yu. Yu. (1969) Discrete programming, ed. D. B. Yudin. Moscow, Nauka.

Medvedev Sergey N. - PhD in Physics and Mathematics, Associate Professor, Department of Computational Mathematics and Applied Information Technologies, Faculty of Applied Mathematics, Informatics, and Mechanics, Voronezh State University.

E-mail: s_n_medvedev@mail.ru

ORCID iD: https://orcid.org/0000-0002-6971-5069 